\title{
Das solitäre adulte Lymphhämangiom der Leber als äußerst seltene Variante einer benignen Raumforderung der Leber
}

Das solitäre adulte Lymphhämangiom der Leber ist ein seltener benigner Tumor, der seit seiner Erstbeschreibung durch Ziegler im Jahr 1892 (Ninard B, Tumor du fois 1959; 453-455) nur in seltenen Fällen dokumentiert wurde. Lymphhämangiome sind in ca. 95\% in der Hals- oder Axillenregion lokalisiert. Die restlichen $5 \%$ verteilen sich auf die übrigen Körperorgane. Ein Befall der Leber ist dabei sehr ungewöhnlich (Stavropoulos M, et al, HPB Surgery 1994; 8: 33-36). Im Folgenden berichten wir über den sehr seltenen Fall eines solitären adulten Lymphhämangioms der Leber als Zufallsbefund bei einem 19jährigen Mann.

\section{Fallbeschreibung}

Im Dezember 2000 befand sich der 19jährige Patient zur Überprüfung der Wehrdienstfähigkeit in der orthopädischen Abteilung des Bundeswehrkrankenhauses Hamburg. Er klagte zusätzlich über einen ungewollten Gewichtsverlust von ca. $20 \mathrm{~kg}$ in den letzten 9 Monaten.

Die eingehende internistische Untersuchung war unauffällig. Auch laborchemisch zeigte sich mit Ausnahme einer geringen Eosinophilie von 8\% (Normwert: $0-4 \%$ ) ein im Wesentlichen unauffälliger Befund.

In der Abdomensonographie fand sich eine subkapsuläre echoreiche Raumforderung im Bereich des rechten Leberlappens. Die Raumforderung stellte sich mit einer Größe von ca. 3,9 cm × 3,2 cm und homogener Binnenstruktur dar. Eine
Vaskularisation war nicht nachweisbar. Ansonsten wurde bei normal großer Leber ein regulärer Sonographiebefund erhoben. Pathologische Lymphknoten oder andere Raumforderungen waren nicht nachweisbar.

Zur weiteren Abklärung erfolgten ein Spiral-CT des Oberbauches in nativer Technik und nach Gabe von $120 \mathrm{ml}$ Imeron $300^{\circledR}$ ein biphasisches Spiral-CT der Leber (arterielle und portale Phase) und einer zusätzlichen späten Phase nach ca. $5 \mathrm{~min}$. Es zeigte sich im rechten Leberlappen im Segment VI eine in nativer Technik hypodense Struktur von ca. 2,0 cm Durchmesser und einer Dichte von -83 HE. Diese Raumforderung ist randständig leicht lobulär konfiguriert, aber scharf abgrenzbar. Septierungen waren nicht ersichtlich. Nach Kontrastmittelgabe konnte ein wesentliches Dichteenhancement nicht nachgewiesen werden. Die übrigen abgebildeten Organstrukturen stellten sich auch in der Computertomographie unauffällig dar.

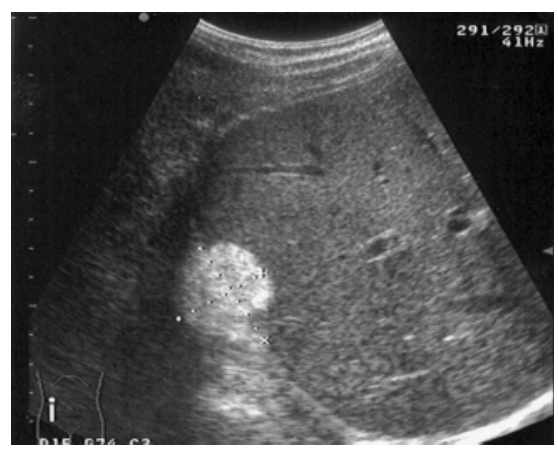

Abb. 1 Sonographiebefund
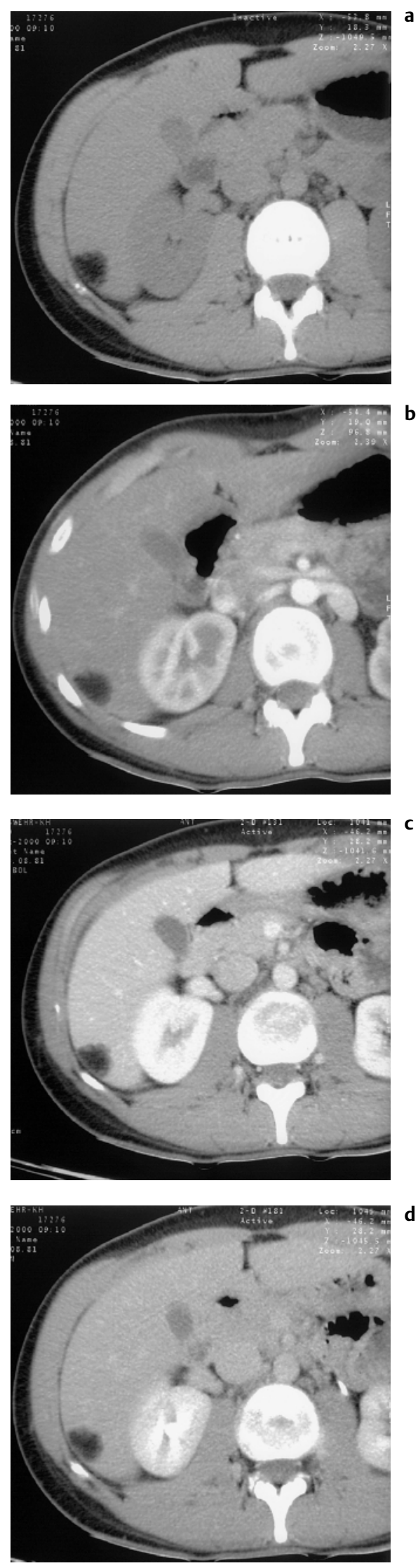

Abb. 2 CT-Befund: (a) nativ, (b) arterielle Phase, (c) portale Phase, (d) urographische Phase 
Aufgrund des Dichteverhaltens wurde ein Fettgewebstumor in der Differenzialdiagnose priorisiert. Da jedoch ein niedrig-malignes Liposarkom nicht sicher auszuschließen war, führten wir eine CT-gesteuerte Punktion des Tumors durch.

Die pathomorphologische Begutachtung ergab als Befund die äußerst rare Variante eines solitären adulten Lymphhämangioms der Leber. Eine lipomatöse Neoplasie konnte immunhistochemisch ausgeschlossen werden.

\section{Diskussion}

Die häufig routinemäßig eingesetzte Sonographie der Oberbauchorgane führt immer wieder zu sonographisch detektierten Leberherden, ohne dass mit dieser Methode eine eindeutige Diagnose zu stellen ist. Von benignen Tumoren wie zum Beispiel Leberhämangiomen oder der fokalen nodulären Hyperplasie (FNH) sind maligne Raumforderungen wie primäre Lebertumoren oder Metastasen zu differenzieren. Zur weiteren Abklärung werden hier zusätzliche bildgebende Verfahren wie die Computertomographie oder die Kernspintomographie eingesetzt. Als Ultima ratio in der Diagnosefindung verbleibt die Probeentnahme, zum Beispiel sonographisch oder computertomographisch gesteuert, zur sicheren histologischen Differenzierung.

Um das Ziel einer möglichst raschen und kostengünstigen Diagnosestellung zu erreichen, müssen bei der Interpretation von bildgebenden Verfahren neben den häufigen Differenzialdiagnosen auch seltene Befunde berücksichtigt werden. Ein solcher seltener, aber differenzialdiagnostisch wichtiger Befund wird in dieser Fallbeschreibung dargestellt.

Das solitäre adulte Lymphhämangiom der Leber ist eine äußerst seltene Variante des vornehmlich in der Hals- oder Axillenregion auftretenden solitären adulten Lymphhämangioms. In nur 5\% der Fälle ist der Befall einer anderen Körperregion nachweisbar. Dieser Tumor ist klinisch unauffällig. Beschwer- den werden nur bei deutlicher Größenzunahme und hierdurch bedingter Verdrängung anderer Organstrukturen angegeben. In der Literatur wird es als benigner Tumor ohne Malignitätspotential beschrieben (Barnes PA, Thomas JL, Bernardino ME, Radiology 1981; 141: 129-133).

Die bildgebenden Verfahren zeigen in den Literaturangaben typischerweise eine zystische oder multizystische Struktur mit Septen, die nur schwerlich von nekrotisierenden Lebermetastasen $\mathrm{zu}$ differenzieren sind (Blumhagen JD, Wood DJ, Rosenbaum DM, J Ultrasound 1987; 6: 487 - 495). Bei den Lymphhämangiomen handelt es sich um meistens angeborene Malformationen des lymphatischen Systems. Die mit Endothel ausgekleideten Höhlen sind mit Lymphe oder chylöser Flüssigkeit gefüllt und führen bei chylösem Inhalt zu negativer Dichte in der Computertomographie. Zur histologischen Differenzierung gegenüber einem in der Struktur sehr ähnlichem Hämangiom der Leber sind der Nachweis von Lymphe oder chylöser Flüssigkeit und das Fehlen von roten Blutkörperchen in den Gängen beweisend. Sonographisch ist eine sichere Differenzierung nicht möglich, da sich beide Strukturen sehr echoreich darstellen. Computertomographisch spricht ein fehlendes Kontrastmittelenhancement gegen ein Hämangiom.

In unserem Falle wurden in der Computertomographie bei fehlendem Kontrastmittelenhancement fettäquivalente Dichtewerte ermittelt, die unsere Differenzialdiagnose zunächst auf einen Fettgewebstumor richtete und die endgültige Diagnose nicht mit einbezog. Nach histologischer Befundung und unserer Literaturrecherche handelt es sich hierbei jedoch um eine benigne Raumforderung der Leber mit der sehr seltenen und, soweit uns bekannt, in nur vier Fällen dokumentierten Diagnose eines solitären adulten Lymphhämangioms der Leber.

M. W. Bartelheimer, H.-M. Schlegel, K. Picolin, Hamburg 